JOURNAL OF

APPLIED

CRYSTALLOGRAPHY

ISSN 1600-5767

\title{
Scattering Methods in Complex Fluids. By Sow-Hsin Chen and Piero Tartaglia. Cambridge University Press, 2015. Pp. 400. Price (hardcover) GBP 75. ISBN 9780521883801.
}

\author{
José Teixeira*
}

Laboratoire Léon Brillouin, CEA Saclay, 91191 Gif-sur-Yvette Cedex, France. * Correspondence e-mail: jose.teixeira@cea.fr

Edited by M. Nespolo, Université de Lorraine, France

Keywords: book reviews; scattering methods; complex fluids

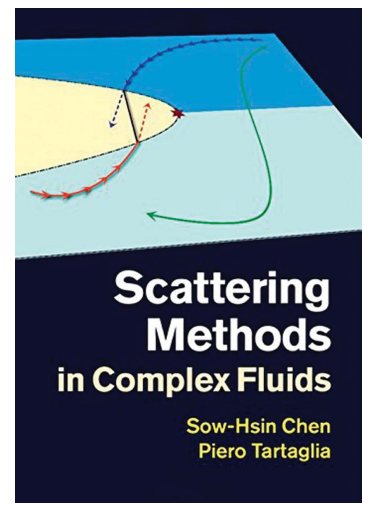

(C) 2015 International Union of Crystallography
In the domain of physical chemistry, many systems exhibit complexities that defy a detailed analysis and establishment of accurate models. Measuring directly the interactions and associated potentials through spatial and time correlation functions at different scales, scattering techniques are among the best methods for the study and eventual characterization of such systems in view of applications. Scattering of electromagnetic radiation or of particles shares a similar theoretical formalism despite the enormous differences between the interactions involved in each case, which are also a source of rich complementarities. Thus, each of these approaches is currently used to study complex fluids.

The book of S.-H. Chen and P. Tartaglia can be seen within this context, despite the fact that a very large part of the text concerns the theory and applications of neutron scattering and, more particularly, small-angle and incoherent quasi-elastic scattering. Also, a large part of the book refers to liquid colloidal solutions, thus covering only a small part of the systems that are normally classified under the denomination of complex fluids. Consequently, the title of the book is a little too wide as compared to its content.

Most of the chapters are divided into 'modules' that are presentations of several specific interesting cases intended to illustrate the theory. This has the advantage of allowing easy and independent reading of some parts. These examples are almost always taken from the remarkable scientific output of the authors or of their close collaborators.

The book is divided into three sections of about 120 pages each. The first one introduces the principles of scattering, focusing more specifically on some details of neutron scattering at small angles, including the processing of raw data (though only for continuous sources). Theories of the structure of simple liquids and aggregates are described in detail as well.

The second section is certainly the more interesting and original part of the book. Under the title Structural arrest, the authors nicely present the mode coupling theory, which applies to viscous liquids, and address the complex issues related to the glass transition. The text is illustrated with examples taken in particular from studies of copolymer solutions. The final chapter of this section deals with sol-gel transition in colloidal solutions.

The last section is, more unexpectedly, entitled Water. Apart from a reference to measurements of the vibrational density of states of the pure liquid presented in the last chapter, the book almost exclusively refers to several samples where water is confined in porous silica or in cement, or is hydrating polymers or DNA. It is likely that the large diversity of situations and of water behaviour in each case explains the classification of this subject under the title of complex fluids. Unlike the presentation of liquid structure in the first part of the book, this section is almost exclusively devoted to the study of the dynamics of water molecules under confinement, relying sometimes on controversial mixture models arising from molecular dynamics simulations.

Despite some heterogeneity, it is an interesting and rich book that would be very useful to all those who intend to revise or deepen their knowledge about the validity of assumptions, approximations and deductions that constitute the background of scattering theories and their limits. It is richly illustrated by numerous figures, taken essentially from publications of the authors. 